\title{
Implantable subcutaneous venous catheters
}

\author{
H P MCDOWELL, C A HART, AND J MARTIN \\ Department of Oncology and Haematology, Alder Hey Children's Hospital, Liverpool
}

\begin{abstract}
SUMmaRY Twelve totally implantable subcutaneous central venous catheter systems were used in paediatric oncology patients. Complications were few-namely, blockage and four infective episodes, all of which were successfully treated. Infective rate was 0.189 episodes per 100 days' use, which is substantially lower than that reported with other central venous catheters.
\end{abstract}

The problems of long term venous access in patients, particularly children, who are receiving prolonged or intensive chemotherapy are well known. Tunnelled central venous catheters were introduced in an attempt to circumvent these problems. ${ }^{12}$ Such catheters are liable to be colonised by skin microorganisms, such as coagulase negative staphylococci, and can result in serious infection, especially in immunocompromised patients. ${ }^{3}$

For the last year a totally implantable subcutaneous central venous catheter and portal with a self sealing septum for hypodermic needle entry (Port-a-Cath) has been employed in managing children with malignant disease. We have analysed our experience, particularly in relation to the number of infective episodes, patient acceptability, and complications necessitating removal.

\section{Materials and method}

The system. The Port-a-Cath system (Pharmacia) consists of a silastic catheter, which is inserted usually through the subclavian vein into the superior vena cava. The catheter is connected distally to a metal reservoir, which has a self sealing septum on the top (Figure). The whole system was implanted subcutaneously under general anaesthesia by one of two surgeons. Access to the system is a sterile procedure, using a Huber point needle, which is pushed through the subcutaneously placed self sealing septum. The system can be used from the day of insertion and was in several of our patients. Maintenance of the system consists of monthly heparinisation, which was carried out by ward staff certified to give intravenous treatment. Before use, the system was flushed with Hepsal (Hepsal, Weddel Pharmaceuticals Ltd), any blood samples that were required were obtained, and then reflushed before connecting to a continuous infusion. The system was used for sampling, to administer chemotherapy, continuously or by bolus, and for delivery of blood or blood products.

Complications. Complications related to the maintenance and use of the systems were recorded both by unit staff and by parents. All febrile episodes, defined as a temperature reading $\geqslant 38^{\circ} \mathrm{C}$, were investigated by blood cultures both from the catheter and a peripheral site. Infections related to the catheter were defined as febrile episodes when micro-organisms were recovered from the catheter or when pus was obtained from the site of insertion of the Port-a-Cath.

\section{Results}

During the study 12 children had a system inserted. Their ages ranged from 1 to 6 years (median 1/1/2 and mean $2 \frac{1}{2}$ years), and they were being treated for acute leukaemia (five cases), neuroblastoma (three), embryonic sarcoma (two), aplastic anaemia (one), and chronic haemolytic anaemia (one). The indications for implanting the system were all children treated in the Oncology Unit at Alder Hey Children's Hospital under 4 years of age who were to receive prolonged chemotherapy; or older children who required frequent venous access whose home circumstances or behaviour favoured a sealed subcutaneous system. There was only one such older child in our study.

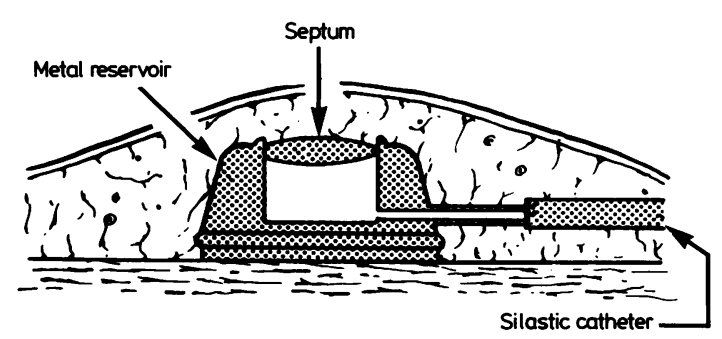

Figure The Port-a-Cath system, showing the inserted silastic catheter connected distally to the metal reservoir, which has a self sealing septum on top. 
To date, catheters have been in situ for a total of 75.75 patient months, ranging from one to 14 months (mean 6.3 months). Each system was in regular use until treatment was completed. Complications have been few. One child developed hypofibrinogenaemia at the time of insertion and subsequently a haemothorax, which required drainage. This was considered to be secondary to her tumour and chemotherapy, rather than due to insertion of the catheter. Blockage in the system was the problem most commonly encountered, occurring in $10 \%$ of infusions, but this was always resolved by flushing or changing the Huber needle. Total blockage requiring removal of the system has not been experienced.

Eighteen febrile episodes occurred during the study, 13 when patients were neutropenic. In four of the episodes micro-organisms were isolated from the catheter and in three of these also from peripheral blood. On three occasions coagulase negative staphylococci were isolated and on one Candida albicans. All were successfully treated with appropriate antibiotics. A focus of infection not related to the catheter was found in five other febrile episodes, and the remainder were apparently noninfective. This represents an infection rate of 0.189 episodes per 100 days of catheter use.

Only one system required removal during use. This child had coagulase negative staphylococcal bacteraemia that was treated successfully. The system required flushing and replacement of the needle on several occasions and was compounded by the need for prolonged and repeated access at the same skin point of entry. Wound sepsis developed over the reservoir with a purulent exudate. On exploration of the chest wall site the reservoir was found to be surrounded by a pool of sterile pus and the system was removed. The line was cultured but was sterile. No other children had wound breakdown, malposition, or breakages.

\section{Discussion}

The use of tunnelled central venous catheters as an effective and safe way of giving prolonged chemotherapy and supportive care in paediatric patients has been advocated. ${ }^{4}$ Such lines, however, are not without their problems.

A recent survey from Bristol described experiences with 49 Broviac or Hickman indwelling central venous catheters inserted in 36 children. ${ }^{5}$ These patients were comparable with ours in terms of age, group of diseases being treated, and chemotherapy used. Twenty nine episodes of infection were encountered and on 21 occasions the catheter was removed for non-elective reasons, each catheter having an average life of 2.9 months. This represents an infection rate of 0.68 episodes per 100 days of catheter use.

The Port-a-Cath system has been developed to circumvent this problem and has been shown to achieve this successfully in our study. The infective episodes were significantly less $(p<0.02)$ compared with that reported by Darbyshire and colleagues $^{5}$ and the necessity to remove systems an uncommon event. Patient acceptability was excellent, discomfort of the wound site only occurring in one patient after insertion, and this settled after three days. Parents were pleased with the cosmetic results. Maintenance of the system is less often required than Broviac or Hickman lines, although the ideal frequency for all systems has not been determined. Two patients in the study had had Broviac catheters inserted already (two and one, respectively), two of which had been pulled out by the patients and the third of which had become infected, reflecting our general difficulties with Broviac and Hickman lines in this very young age group. We, therefore, routinely insert Port-a-Cath systems in young children less than 4 years who require prolonged venous access.

\section{References \\ 1 Broviac JW, Cole JJ, Scribner BH. A silicone rubber atrial catheter for prolonged parenteral nutrition. Surg Gynecol Obstet 1973;136:602-6. \\ ${ }^{2}$ Hickman RO, Buckner CD, Clift RA, Sanders JE, Stewart P, Thomas ED. A modified right atrial catheter for access to the venous system in marrow transplant recipients. Surg Gynecol Obstet 1979;148:871-5. \\ 3 Wade JC, Schimpff SC, Newman KA, Wiernick PH. Staphylo- coccus epidermis: an increasing cause of infection in patients with granulocytopaenia. Ann Intern Med; 97:503-8. \\ ${ }^{4}$ Bothe A, Piccione W, Ambrosino J, Benotti P, Lokich J. Implantable central venous access system. Am $J$ Surg 1984;147:565-9. \\ ${ }^{5}$ Darbyshire PJ, Weightman NC, Speller DCE. Problems associ- ated with indwelling central venous catheters. Arch Dis Child 1985;60:129-34.}

Correspondence to Dr H McDowell, Oncology Unit, Alder Hey Children's Hospital, Eaton Road, Liverpool L12 2AP, England.

Received 16 June 1986 